\title{
ATTRACTOR BIFURCATION OF THREE-DIMENSIONAL DOUBLE-DIFFUSIVE CONVECTION
}

\author{
CHUN-HSIUNG HSIA, TIAN MA, AND SHOUHONG WANG
}

\begin{abstract}
In this article, we present a bifurcation analysis on the doublediffusive convection. Two pattern selections, rectangles and squares, are investigated. It is proved that there are two different types of attractor bifurcations depending on the thermal and salinity Rayleigh numbers for each pattern. The analysis is based on a newly developed attractor bifurcation theory, together with eigen-analysis and the center manifold reductions.
\end{abstract}

\section{INTRODUCTION}

The main objective of this article is to develop a bifurcation and stability theory for the double-diffusive convection, using a newly developed bifurcation theory for dynamical systems, both finite and infinite dimensional [11. Convective motions occur in a fluid when there are density variations present. Double-diffusive convection is the name given to such convective motions when the density variations are caused by two different components which have different rates of diffusion. Doublediffusion was first originally discovered in the 1857 by Jevons 9 , forgotten, and then rediscovered as an "oceanographic curiosity" a century later; see among others Stommel, Arons and Blanchard [17, Veronis [19, and Baines and Gill [1.

We have conducted a bifurcation and stability analysis in 8 for the two dimensional (2D) double-diffusive convection model. In this article, we continue our analysis to study the three dimensional (3D) double-diffusive convection problem. As in the two-dimensional case, the governing equations are the Boussinesq equations with two diffusion equations of the temperature and salinity functions.

The double-diffusive system involves four important nondimensional parameters: the thermal Rayleigh number $\lambda$, the solute Rayleigh number $\eta$, the Prandtl number $\sigma$ and the Lewis number $\tau$. We examine in this article different transition/instability regimes defined by these parameters. We study the onset of double diffusive instabilities in these regimes. In the ocean circulation case, the Prandtl and Lewis numbers satisfy $\sigma>1$ and $\tau<1$, as the heat diffuses about 100 times more rapidly than salt [16. In this case, different regimes of stabilities and instabilities/transitions of the basic state can be described by regions in the $\lambda-\eta$ plane (the thermal and salt Rayleigh numbers) as shown in Figure 3.1. In this article, we focus on the regimes where $\eta<\eta_{c}$; see Figure 3.1. In the case where $\eta>\eta_{c}$, transitions to periodic or aperiodic solutions are expected, and will be addressed elsewhere.

Date: September 4, 2018.

The work was supported in part by the Office of Naval Research, by the National Science Foundation, and by the National Science Foundation of China. 
In both $2 \mathrm{D}$ and $3 \mathrm{D}$ cases, the central gravity of the analysis is the reduction of the problem to the center manifold in the first unstable eigendirections, based on an approximation formula for the center manifold function. The key idea is to find the approximation of the reduction to certain order, leading to a "nondegenerate" system with higher order perturbations. The analysis for the 3D case studied here has all the difficulties appeared in the analysis for the $2 \mathrm{D}$ case such as the nonsymmetric linearized eigenvalue problem. Additional difficulties occur in the 3D case. One difficulty is the lack of the existence of global strong solutions of the 3D Boussinesq equations, and another is the higher dimensionality of the generalized eigenspace, leading to the center manifold reduction to higher dimensional center manifolds, and consequently to more complicated dynamics.

With this reduction in our disposal, a general bifurcation theorem follows from the general strategy of attractor bifurcations. We prove in particular that there are two different types of transition/bifurcation from the basic state: one is subcritical and the other is supercritical. In the rectangle case (i.e. the horizontal domain is a rectangle), the types of transition/bifurcation are dictated explicitly by a nondimensional parameter $\eta_{c_{1}}$; see Figure 3.1. In the square case (i.e. the horizontal domain is a square), types are determined by the quadratic form (3.11). From the physical point of view, the supercritical bifurcation corresponds to a continuous transition. The subcritical bifurcation corresponds, however, to a jump transition, a very different transition. As demonstrated in the $2 \mathrm{D}$ case [8], this subcritical bifurcation leads to the existence of a saddle-node bifurcation and the hysteresis phenomena, thanks to the existence of strong solutions and the existence of global attractors. In the 3D case studied here in this article, we are not able to make such conclusions. However, we can still prove that there is an absorbing region resembling the main properties of the saddle-node bifurcation and hysteresis.

There have been extensive studies on bifurcation and stability analysis for convection models; see among others [14, 2, 3, 6] and the references therein. We mention in particular that in [3], Buzano and Golubitsky used the singularity-theory method to study the problem of pattern formation as it relates to bifurcation with respect to the hexagonal lattice. They treated bifurcation problems that are symmetric with respect to group preserving doubly periodic functions on a hexagonal lattice. Their method provides a delicate alternative to study the convection problem in this paper.

Another remark is that as mentioned by Sattinger on page 96 in [15], a stable bifurcating solution with respect to a subclass of disturbances of full symmetry might turn out to be unstable with respect to the disturbances of full symmetry. In our analysis, although we use the symmetry properties implicitly to reduce the calculations, we don't use the equivariant theory to reduce the problem to a lower dimensional space. Since we want to keep as much stability information as possible.

This article is organized as follows. The basic governing equations are given in Section 2. The main theorems are stated in Section 3. The remaining sections are devoted to the proof of the main theorems, with Section 4 on eigenvalue problems, Section 5 on center manifold reductions and the proofs of the theorems. 


\section{The Double-Diffusive Equations}

The nondimensional double-diffusive convection problem in a three-dimensional (3D) domain $\Omega=\mathbb{R}^{2} \times(0,1) \subset \mathbb{R}^{3}$ with coordinates denoted by $(x, y, z)$ are given as follows; see Veronis [19]:

$$
\left\{\begin{array}{l}
\frac{\partial U}{\partial t}=\sigma(\Delta U-\nabla p)+\sigma(\lambda T-\eta S) e-(U \cdot \nabla) U, \\
\frac{\partial T}{\partial t}=\Delta T+w-(U \cdot \nabla) T \\
\frac{\partial S}{\partial t}=\tau \Delta S+w-(U \cdot \nabla) S, \\
\operatorname{div} U=0
\end{array}\right.
$$

where $U=(u, v, w), \lambda$ the thermal Rayleigh number, $\eta$ the salinity Rayleigh number, $\sigma$ the Prandtl number, and $\tau$ the Lewis number. We consider the periodic boundary condition in the $x$ and $y$ directions

$$
\begin{aligned}
(U, T, S)(x, y, z, t) & =(U, T, S)\left(x+2 j \pi / \alpha_{1}, y, z, t\right) \\
& =(U, T, S)\left(x, y+2 k \pi / \alpha_{2}, z, t\right),
\end{aligned}
$$

for any $j, k \in \mathbb{Z}$. At the top and bottom boundaries, we impose the free-free boundary conditions; namely,

$$
(T, S, w)=0, \quad \frac{\partial u}{\partial z}=0, \quad \frac{\partial v}{\partial z}=0, \quad \text { at } \quad z=0,1 .
$$

It's natural to put the constraint

$$
\int_{\Omega} u d x d y d z=\int_{\Omega} v d x d y d z=0
$$

for the problem (2.1)-(2.3). It is easy to see that (2.1) is invariant under this constraint. The initial value conditions are given by

$$
(U, T, S)=(\widetilde{U}, \widetilde{T}, \widetilde{S}) \quad \text { at } \quad t=0 .
$$

Let

$$
\begin{aligned}
H & =\left\{(U, T, S) \in L^{2}(\Omega)^{5}|\operatorname{div} U=0, w|_{z=0,1}=0,(u, v) \text { satisfy (2.2) and (2.4) }\right\}, \\
H_{1} & =\left\{(U, T, S) \in H^{2}(\Omega)^{5} \cap H \mid(U, T, S) \text { satisfies (2.2) and (2.3) }\right\} .
\end{aligned}
$$

Let $G: H_{1} \rightarrow H$, and $L_{\lambda \eta}=-A-B_{\lambda \eta}: H_{1} \rightarrow H$ be defined by

$$
\begin{aligned}
& G(\psi)=(-P[(U \cdot \nabla) U],-(U \cdot \nabla) T,-(U \cdot \nabla) S), \\
& A \psi=(-P[\sigma(\Delta U)],-\Delta T,-\tau \Delta S), \\
& B_{\lambda \eta} \psi=(-P[\sigma(\lambda T-\eta S) e],-w,-w),
\end{aligned}
$$

for any $\psi=(U, T, S) \in H_{1}$. Here $P$ is the Leray projection to $L^{2}$ fields, and for a detailed account of the function spaces, see among many others [18. Then the Boussinesq equations (2.1)-(2.4) can be written in the following operator form

$$
\frac{d \psi}{d t}=L_{\lambda \eta} \psi+G(\psi), \quad \psi=(U, T, S)
$$

It is classical that there is a global weak solution for the system, and there is a global strong solution for small data; see among others [5, 10. Of course, the global existence of strong solutions is not known for large data. 


\section{Main Results}

3.1. Attractor bifurcation theory. In this subsection, we recapitulate the attractor bifurcation theory introduced by two of the authors in [12, 11.

Let $H$ and $H_{1}$ be two Hilbert spaces, and $H_{1} \hookrightarrow H$ be a dense and compact inclusion. We consider the following nonlinear evolution equations

$$
\left\{\begin{array}{l}
\frac{d u}{d t}=L_{\lambda} u+G(u, \lambda), \\
u(0)=u_{0},
\end{array}\right.
$$

where $u:[0, \infty) \rightarrow H$ is the unknown function, $\lambda \in \mathbb{R}$ is the system parameter, and $L_{\lambda}: H_{1} \rightarrow H$ are parameterized linear completely continuous fields depending continuously on $\lambda \in \mathbb{R}^{1}$, which satisfy

$$
\begin{cases}-L_{\lambda}=A+B_{\lambda} & \text { a sectorial operator, } \\ A: H_{1} \rightarrow H & \text { a linear homeomorphism, } \\ B_{\lambda}: H_{1} \rightarrow H & \text { parameterized linear compact operators. }\end{cases}
$$

It is easy to see [7] that $L_{\lambda}$ generates an analytic semi-group $\left\{e^{t L_{\lambda}}\right\}_{t \geq 0}$. Then we can define fractional power operators $\left(-L_{\lambda}\right)^{\mu}$ for any $0 \leq \mu \leq 1$ with domain $H_{\mu}=D\left(\left(-L_{\lambda}\right)^{\mu}\right)$ such that $H_{\mu_{1}} \subset H_{\mu_{2}}$ if $\mu_{1}>\mu_{2}$, and $H_{0}=H$.

Furthermore, we assume that the nonlinear terms $G(\cdot, \lambda): H_{\mu} \rightarrow H$ for some $1>\mu \geq 0$ are a family of parameterized $C^{r}$ bounded operators $(r \geq 1)$ continuously depending on the parameter $\lambda \in \mathbb{R}^{1}$, such that

$$
G(u, \lambda)=o\left(\|u\|_{H_{\mu}}\right), \quad \forall \lambda \in \mathbb{R}^{1} .
$$

In this paper, we are interested in the sectorial operator $-L_{\lambda}=A+B_{\lambda}$ such that there exist an eigenvalue sequence $\left\{\rho_{k}\right\} \subset \mathbb{C}^{1}$ and an eigenvector sequence $\left\{e_{k}, h_{k}\right\} \subset H_{1}$ of $A$ :

$$
\left\{\begin{array}{l}
A z_{k}=\rho_{k} z_{k}, \quad z_{k}=e_{k}+i h_{k}, \\
\operatorname{Re} \rho_{k} \rightarrow \infty(k \rightarrow \infty) \\
\left|\operatorname{Im} \rho_{k} /\left(a+\operatorname{Re} \rho_{k}\right)\right| \leq c
\end{array}\right.
$$

for some $a, c>0$, such that $\left\{e_{k}, h_{k}\right\}$ is a basis of $H$. Also we assume that there is a constant $0<\theta<1$ such that

$$
B_{\lambda}: H_{\theta} \longrightarrow H \text { bounded, } \forall \lambda \in \mathbb{R}^{1} \text {. }
$$

Under conditions (3.4) and (3.5), the operator $-L_{\lambda}=A+B_{\lambda}$ is a sectorial operator.

Let $\left\{S_{\lambda}(t)\right\}_{t \geq 0}$ be an operator semi-group generated by the equation (3.1). Then the solution of (3.1) can be expressed as $\psi\left(t, \psi_{0}\right)=S_{\lambda}(t) \psi_{0}, \quad t \geq 0$.

Consider (3.1) satisfying (3.2) and (3.3). We start with the Principle of Exchange of Stabilities (PES). Let the eigenvalues (counting the multiplicity) of $L_{\lambda}$ be given by $\beta_{1}(\lambda), \beta_{2}(\lambda), \cdots$. Suppose that

$$
\begin{gathered}
\operatorname{Re} \beta_{i}(\lambda)\left\{\begin{array}{ll}
<0, & \text { if } \lambda<\lambda_{0} \\
=0, & \text { if } \lambda=\lambda_{0} \\
>0, & \text { if } \lambda>\lambda_{0}
\end{array} \quad(1 \leq i \leq m)\right. \\
\operatorname{Re} \beta_{j}\left(\lambda_{0}\right)<0 . \quad \forall m+1 \leq j .
\end{gathered}
$$


Let the eigenspace of $L_{\lambda}$ at $\lambda_{0}$ be

$$
E_{0}=\bigcup_{1 \leq j \leq m} \bigcup_{k=1}^{\infty}\left\{u, v \in H_{1} \mid\left(L_{\lambda_{0}}-\beta_{j}\left(\lambda_{0}\right)\right)^{k} w=0, w=u+i v\right\} .
$$

It is known that $\operatorname{dim} E_{0}=m$.

Theorem 3.1 (T. Ma and S. Wang [12, 11]). Assume that the conditions (3.2)(3.5) and (3.6)-(3.7) hold true, and $u=0$ is locally asymptotically stable for (3.1) at $\lambda=\lambda_{0}$. Then the following assertions hold true.

(1) (3.1) bifurcates from $(u, \lambda)=\left(0, \lambda_{0}\right)$ to attractors $\Sigma_{\lambda}$, having the same homology as $S^{m-1}$, for $\lambda>\lambda_{0}$, with $m-1 \leq \operatorname{dim} \Sigma_{\lambda} \leq m$, which is connected if $m>1$;

(2) For any $u_{\lambda} \in \Sigma_{\lambda}, u_{\lambda}$ can be expressed as

$$
u_{\lambda}=v_{\lambda}+o\left(\left\|v_{\lambda}\right\|_{H_{1}}\right), v_{\lambda} \in E_{0}
$$

(3) There is an open set $U \subset H$ with $0 \in U$ such that the attractor $\Sigma_{\lambda}$ bifurcated from $\left(0, \lambda_{0}\right)$ attracts $U \backslash \Gamma$ in $H$, where $\Gamma$ is the stable manifold of $u=0$ with co-dimension $m$.

3.2. Main theorems. We now consider the double diffusive convection equations (2.1). In this article, we always consider the case where the parameters $\lambda$ and $\eta$ satisfy

$$
\begin{aligned}
& \eta<\eta_{c}=\frac{27}{4} \pi^{4} \tau^{2}\left(1+\sigma^{-1}\right)(1-\tau)^{-1}, \\
& \lambda \approx \lambda_{c}=\frac{\eta}{\tau}+\frac{27}{4} \pi^{4} .
\end{aligned}
$$

Two pattern selections, rectangles and squares, are studied.

3.2.1. Rectangle Case. For the rectangle case, we assume that

$$
\alpha_{1}=\frac{\pi}{\sqrt{2}} \quad \text { and } \quad \alpha_{2} \neq \alpha_{1} .
$$

Here the condition on $\alpha_{1}$ and $\alpha_{2}$ defines the aspect ratios of the domain. The first eigenspace of the eigenvalue problem (4.1) is of dimension two.

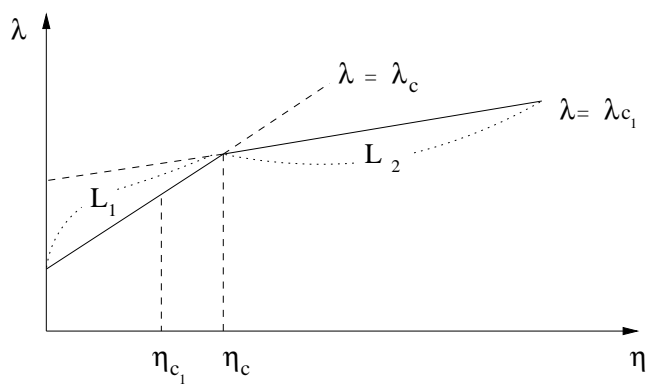

FiguRE 3.1.

First we consider a more physically relevant diffusive regime where the thermal Prandtl number $\sigma$ is bigger than 1, and the Lewis number $\tau$ is less than 1:

$$
\sigma>1>\tau \text {. }
$$


In this case, we consider two straight lines in the $\lambda-\eta$ parameter plane as shown in Figure 3.1

$$
\begin{cases}L_{1}: & \lambda=\lambda_{c}(\eta)=\frac{\eta}{\tau}+\frac{27}{4} \pi^{4}, \\ L_{2}: & \lambda=\lambda_{c_{1}}(\eta)=\frac{(\sigma+\tau)}{(\sigma+1)} \eta+\frac{27}{4} \pi^{4}\left(1+\sigma^{-1} \tau\right)(1+\tau) .\end{cases}
$$

Also shown in Figure 3.1 are two critical values for $\eta$ given by

$$
\eta_{c}=\frac{27}{4} \pi^{4} \tau^{2}\left(1+\sigma^{-1}\right)(1-\tau)^{-1}, \quad \eta_{c_{1}}=\frac{27}{4} \pi^{4} \tau^{3}\left(1-\tau^{2}\right)^{-1} .
$$

The following two theorems study the transitions/bifurcation of the doublediffusive model near the line $L_{1}$ for $\eta<\eta_{c}$.

Theorem 3.2. Assume that the condition (3.8) holds true, $\sigma>1>\tau$, and $\eta<$ $\eta_{c_{1}}=\frac{27}{4} \pi^{4} \tau^{3}\left(1-\tau^{2}\right)^{-1}$. Then the following assertions for the problem (2.1)-2.4) hold true.

(1) If $\lambda \leq \lambda_{c}$, the steady state $(U, T, S)=0$ is locally asymptotically stable for the problem.

(2) For $\lambda>\lambda_{c}$, the solutions bifurcate from $((U, T, S), \lambda)=\left(0, \lambda_{c}\right)$ to an attractor $\Sigma_{\lambda}=S^{1}$, consisting of steady state solutions of the problem.

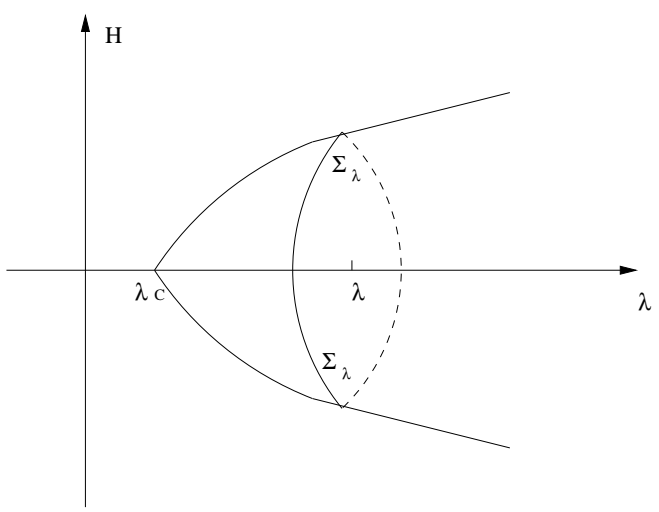

FiguRE 3.2. If $\tau>1$ or $\eta<\eta_{c_{1}}$, the solutions bifurcate from $\left(0, \lambda_{c}\right)$ to an attractor $\Sigma_{\lambda}$ for $\lambda>\lambda_{c}$.

Theorem 3.3. Assume that the condition (3.8) holds true, $\sigma>1>\tau$ and $\eta_{c}>\eta>$ $\eta_{c_{1}}=\frac{27}{4} \pi^{4} \tau^{3}\left(1-\tau^{2}\right)^{-1}$. Then on $\lambda<\lambda_{c}$, the problem (2.1)- 2.4) bifurcates from $((U, T, S), \lambda)=\left(0, \lambda_{c}\right)$ to a repeller $\Sigma_{\lambda}=S^{1}$, consisting of steady state solutions of the problem. 


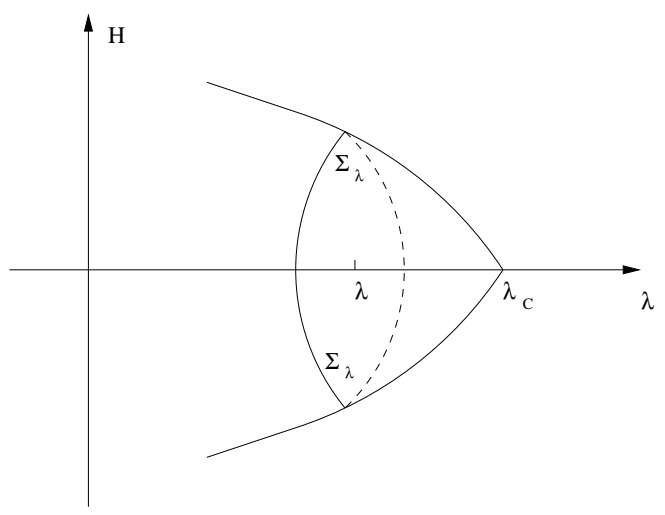

FiguRE 3.3. If $\tau<1$ and $\eta_{c_{1}}<\eta<\eta_{c}$, the problem has a subcritical type bifurcation for $\lambda<\lambda_{c}$.

We now consider the diffusive parameter regime where $\sigma>1, \tau>1$, and $\sigma \neq \tau$. In this case, two lines are shown in Figure 3.4. The following theorem provides bifurcation when $\lambda$ crosses the line $L_{1}$.

Theorem 3.4. Assume that $\sigma>1, \tau>1, \sigma \neq \tau$ and (3.8) hold true, then for any $\eta>0$, the following assertions for the problem (2.1)- (2.4) hold true.

(1) If $\lambda \leq \lambda_{c}$, the steady state $(U, T, S)=0$ is locally asymptotically stable for the problem.

(2) For $\lambda>\lambda_{c}$, the solutions bifurcate from $((U, T, S), \lambda)=\left(0, \lambda_{c}\right)$ to an attractor $\Sigma_{\lambda}$, homeomorphic to $S^{1}$, which consists of steady state solutions of the problem.

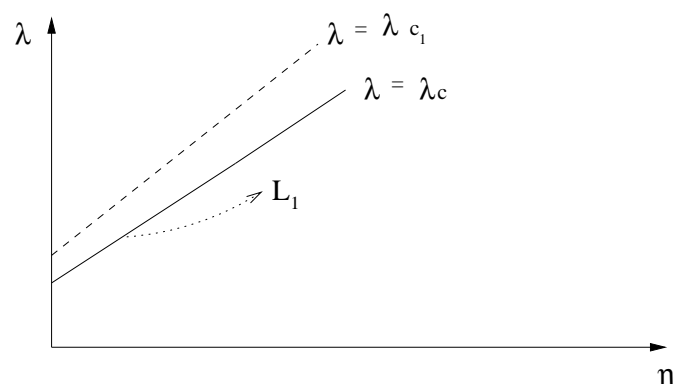

FigURE 3.4 .

3.2.2. Square Case. For the square case, we assume $\alpha_{1}=\alpha_{2}=\frac{\pi}{2}$. Then, the first eigenspace of the eigenvalue problem (4.1) is of dimension 4. In this case, the bifurcation type is determined by the following quadratic form

(3.11) $\delta_{1}\left(x_{1111}^{2}+y_{1111}^{2}+x_{1-111}^{2}+y_{1-111}^{2}\right)^{2}+2 \delta_{2}\left(x_{1111}^{2}+y_{1111}^{2}\right)\left(x_{1-111}^{2}+y_{1-111}^{2}\right)$, where $\delta_{1}$ and $\delta_{2}$ are as defined in (5.7) and (5.8), which can be evaluated precisely when the parameters $\tau, \sigma, \eta$ and $\lambda$ are given. More precisely, we have the following two theorems. 
Theorem 3.5. Assume that (3.8) holds true and (3.11) is negative definite, then the following assertions for the problems (2.1)-2.4) hold true.

(1) If $\lambda \leq \lambda_{c}$, the steady state $(U, T, S)=0$ is locally asymptotically stable for the problem.

(2) For $\lambda>\lambda_{c}$, the solutions bifurcate from $((U, T, S), \lambda)=\left(0, \lambda_{c}\right)$ to an attractor $\Sigma_{\lambda}$, having the same homology as $S^{3}$.

Theorem 3.6. Assume that (3.8) holds true and (3.11) is positive definite. Then on $\lambda<\lambda_{c}$, the problem (2.1)-2.4) bifurcates from $((U, T, S), \lambda)=\left(0, \lambda_{c}\right)$ to a repeller $\Sigma_{\lambda}$, having the same homology as $S^{3}$.

\section{Eigenvalue PRoblem}

In order to apply the center manifold theory to reduce the bifurcation problems, we consider the following eigenvalue problem for the linearized equations of (2.1)(2.4):

$$
\left\{\begin{array}{l}
\sigma(\Delta U-\nabla p)+\sigma(\lambda T-\eta S) e=\beta U, \\
\Delta T+w=\beta T \\
\tau \Delta S+w=\beta S, \\
\operatorname{div} U=0
\end{array}\right.
$$

supplemented with (2.3) and (2.4).

4.1. Eigenvalues. We shall use the method of separation of variables to deal with the problem (4.1). Since $\psi=(U, T, S)$ satisfies the periodic condition (2.2), we expand the fields in Fourier series as

$$
\psi(x, y, z)=\sum_{j, k=-\infty}^{\infty} \psi_{j k}(z) e^{i\left(j \alpha_{1} x+k \alpha_{2} y\right)} .
$$

Plugging (4.2) into (4.1), we obtain the following ODE system:

$$
\left\{\begin{array}{l}
D_{j k} u_{j k}-i j \alpha_{1} p_{j k}=\sigma^{-1} \beta u_{j k}, \\
D_{j k} v_{j k}-i k \alpha_{2} p_{j k}=\sigma^{-1} \beta v_{j k}, \\
D_{j k} w_{j k}-p_{j k}^{\prime}+\lambda T_{j k}-\eta S_{j k}=\sigma^{-1} \beta w_{j k}, \\
D_{j k} T_{j k}+w_{j k}=\beta T_{j k}, \\
\tau D_{j k} S_{j k}+w_{j k}=\beta S_{j k}, \\
i j \alpha_{1} u_{j k}+i k \alpha_{2} v_{j k}+w_{j k}^{\prime}=0, \\
\left.u_{j k}^{\prime}\right|_{z=0,1}=\left.v_{j k}^{\prime}\right|_{z=0,1}=\left.w_{j k}\right|_{z=0,1}=\left.T_{j k}\right|_{z=0,1}=\left.S_{j k}\right|_{z=0,1}=0,
\end{array}\right.
$$

for $j, k \in \mathbb{Z}$, where ${ }^{\prime}=d / d z, D_{j k}=d^{2} / d z^{2}-\alpha_{j k}^{2}$ and $\alpha_{j k}^{2}=j^{2} \alpha_{1}^{2}+k^{2} \alpha_{2}^{2}$. If $w_{j k} \neq 0$, (4.3) can be reduced to a single equation for $w_{j k}(z)$ :

$$
\begin{aligned}
& \left\{\left(\tau D_{j k}-\beta\right)\left(D_{j k}-\beta\right)\left(D_{j k}-\sigma^{-1} \beta\right) D_{j k}\right. \\
& \left.\quad+\alpha_{j k}^{2}\left[\lambda\left(\tau D_{j k}-\beta\right)-\eta\left(D_{j k}-\beta\right)\right]\right\} w_{j k}=0, \\
& w_{j k}=w_{j k}^{\prime \prime}=w_{j k}^{(4)}=w_{j k}^{(6)}=0 \quad \text { at } \quad z=0,1,
\end{aligned}
$$


for $j, k \in \mathbb{Z}$. Thanks to (4.5), $w_{j k}$ can be expanded in a Fourier sine series

$$
w_{j k}(z)=\sum_{l=1}^{\infty} w_{j k l} \sin l \pi z
$$

for $(j, k) \in \mathbb{Z} \times \mathbb{Z}$. Substituting (4.6) into (4.4), we see that the corresponding eigenvalues $\beta$ of Problem (4.1) satisfy the cubic equations

$$
\begin{gathered}
\beta^{3}+(\sigma+\tau+1) \gamma_{j k l}^{2} \beta^{2}+\left[(\sigma+\tau+\sigma \tau) \gamma_{j k l}^{4}-\sigma \alpha_{j k}^{2} \gamma_{j k l}^{-2}(\lambda-\eta)\right] \beta \\
+\sigma \tau \gamma_{j k l}^{6}+\sigma \alpha_{j k}^{2}(\eta-\tau \lambda)=0,
\end{gathered}
$$

for $j, k \in \mathbb{Z}$ and $l \in \mathbb{N}$, where $\gamma_{j k l}^{2}=\alpha_{j k}^{2}+l^{2} \pi^{2}$.

For the sake of convenience to analyze the distribution of the eigenvalues, we now introduce some notations. For fixed parameters $\sigma, \tau, \eta$ and $\lambda$, let

$$
\begin{aligned}
& g_{j k l}(\beta)=\beta^{3}+(\sigma+\tau+1) \gamma_{j k l}^{2} \beta^{2}+(\sigma+\tau+\sigma \tau) \gamma_{j k l}^{4} \beta+\sigma \tau \gamma_{j k l}^{6}, \\
& h_{j k l}(\beta)=\left[\sigma \alpha_{j k}^{2} \gamma_{j k l}^{-2}(\lambda-\eta)\right] \beta-\sigma \alpha_{j k}^{2}(\eta-\tau \lambda), \\
& f_{j k l}(\beta)=g_{j k l}(\beta)-h_{j k l}(\beta), \quad \eta_{c}=\frac{27}{4} \pi^{4} \tau^{2}\left(1+\sigma^{-1}\right)(1-\tau)^{-1}, \\
& \eta_{c_{1}}=\frac{27}{4} \pi^{4} \tau^{3}\left(1-\tau^{2}\right)^{-1}, \quad \lambda_{c}=\frac{\eta}{\tau}+\frac{27}{4} \pi^{4} .
\end{aligned}
$$

Furthermore, let $\beta_{j k l 1}, \beta_{j k l 2}$ and $\beta_{j k l 3}$ be the zeros of $f_{j k l}$ with

$$
\operatorname{Re}\left(\beta_{j k l 1}\right) \geq \operatorname{Re}\left(\beta_{j k l 2}\right) \geq \operatorname{Re}\left(\beta_{j k l 3}\right),
$$

and let $\beta_{j k l \sigma}=-\sigma \gamma_{j k l}^{2}$.

The following lemma characterizes the eigenvectors of $L_{\lambda \eta}$ with zero $w$-components; the proof is straightforward and we omit the details.

Lemma 4.1. For $l \in 0 \cup \mathbb{N}$ and $(j, k) \in \mathbb{Z}^{2}, \beta_{j k l \sigma}$ is an eigenvalue of the problem (4.1). Moreover, the following assertions are true:

(1) If $(j, k)=(0,0)$ and $l>0$, the corresponding eigenvectors are

$$
\psi_{1}^{\beta_{00 l \sigma}}=(\cos l \pi z, 0,0,0,0)^{t}, \quad \psi_{2}^{\beta_{00 l \sigma}}=(0, \cos l \pi z, 0,0,0)^{t} ;
$$

(2) If $j^{2}+k^{2} \neq 0$ and $l \in 0 \cup \mathbb{N}$, the corresponding eigenvectors are

$$
\begin{aligned}
& \psi_{1}^{\beta_{j k l \sigma}}=\left(k \cos \left(j \alpha_{1} x+k \alpha_{2} y\right) \cos l \pi z,-j \cos \left(j \alpha_{1} x+k \alpha_{2} y\right) \cos l \pi z, 0,0,0\right)^{t}, \\
& \psi_{2}^{\beta_{j k l \sigma}}=\left(k \sin \left(j \alpha_{1} x+k \alpha_{2} y\right) \cos l \pi z,-j \sin \left(j \alpha_{1} x+k \alpha_{2} y\right) \cos l \pi z, 0,0,0\right)^{t} .
\end{aligned}
$$

In the following discussions, we shall focus on the following diffusive regime:

$$
\sigma>1>\tau>0, \eta<\eta_{c} \text { and } \lambda \approx \lambda_{c} .
$$

Lemma 4.2. Suppose that $\alpha_{j k}^{2}=\frac{\pi^{2}}{2}$ and 4.10) holds true, then $f_{j k 1}(\beta)$ has three simple real zeros.

Proof. Since $\lambda \approx \lambda_{c}$, it suffices to prove this statement for $\lambda=\lambda_{c}$. In this case,

$$
\begin{aligned}
f_{j k 1}(\beta) & =\beta^{3}+\left[3 \pi^{2}(\sigma+\tau+1) / 2\right] \beta^{2}+\left[9 \pi^{4}(\sigma+\tau+\sigma \tau) / 4-\sigma\left(\lambda_{c}-\eta\right) / 3\right] \beta \\
& =f(\beta) \beta .
\end{aligned}
$$

Since $\eta<\eta_{c}$, the constant term of $f(\beta)$ is positive. Hence $\beta_{j k 11}=0$ is a simple zero of $f_{j k 1}$. Moreover, the quadratic discriminant of $f(\beta)$ is $9 \pi^{4}(\sigma+1-\tau)^{2} / 4+$ $4 \sigma \eta(1-\tau) /(3 \tau)>0$. This implies $f_{j k 1}$ has three simple real zeros. 
We summarize the following important lemma about the distribution of the zeros of $f_{j k l}$. From the physical point of view, this lemma verifies the principle of exchange of stabilities (PES).

Lemma 4.3. Assume that either 1) $0<\eta<\eta_{c}$ with $\tau<1$ or 2) $\eta>0$ with $\tau>1$ holds true, then

$$
\begin{array}{ll}
\beta_{j k 11}(\lambda) \begin{cases}<0 & \text { if } \lambda<\lambda_{c} \\
=0 & \text { if } \lambda=\lambda_{c} \\
>0 & \text { if } \lambda>\lambda_{c}\end{cases} & \text { if } \alpha_{j k}^{2}=\frac{\pi^{2}}{2}, \\
\operatorname{Re} \beta_{j k l q}(\lambda)<0 & \text { if }\left(\alpha_{j k}^{2}, l, q\right) \neq\left(\frac{\pi^{2}}{2}, 1,1\right) .
\end{array}
$$

Before proceeding to the proof, we make a few remarks as follows.

Remark 4.4. The distribution of the zeros of $f_{j k l}$ was first partially analyzed by Veronis [19; see also P.G. Baines [1. The complete proof of this lemma is similar to the $2 \mathrm{D}$ case in 8 , we shall omit it.

To check that the operators $-L_{\lambda \eta}$ satisfy condition (3.4), we prove the following lemma.

Lemma 4.5. (1) All but finitely many zeros of $f_{j k l}(\beta)$ are negative real numbers for $(j, k, l) \in \mathbb{Z}^{2} \times \mathbb{N}$.

(2) $\beta_{j k l q} \rightarrow-\infty$ if $j^{2}+k^{2}+l^{2} \rightarrow \infty$.

Proof. Since $f_{j k l}=g_{j k l}-h_{j k l}, \beta$ is a zero of $f_{j k l}(\beta)$ if and only if $\beta$ satisfies the equation

$$
g_{j k l}(\beta)=h_{j k l}(\beta) .
$$

Plugging $\beta=\gamma_{j k l}^{2} \beta^{*}$ into (4.13), we obtain

$$
\left(\beta^{*}+1\right)\left(\beta^{*}+\tau\right)\left(\beta^{*}+\sigma\right)=\vartheta_{j k l}\left[(\lambda-\eta) \beta^{*}-(\eta-\tau \lambda)\right],
$$

where $\vartheta_{j k l}=\sigma \alpha_{j k}^{2} / \gamma_{j k l}^{6}$. Since $\lim _{j^{2}+k^{2}+l^{2} \rightarrow \infty} \vartheta_{j k l}=0$, the roots of (4.14) must be negative real numbers near the interval $[-\sigma,-\tau]$ when $\left(j^{2}+k^{2}+l^{2}\right)$ is large. This completes the proof.

4.2. Eigenvectors. We now make some observations to analyze the spectrum of $L_{\lambda \eta}$. Since $g_{j k l}(\beta)=\left(\beta+\gamma_{j k l}^{2}\right)\left(\beta+\tau \gamma_{j k l}^{2}\right)\left(\beta+\sigma \gamma_{j k l}^{2}\right)$ and $h_{j k l}=\sigma \alpha_{j k}^{2} \gamma_{j k l}^{-2}[(\lambda-$ $\left.\eta) \beta-(\eta-\tau \lambda) \gamma_{j k l}^{2}\right]$, it's easy to check that $\beta=-\gamma_{j k l}^{2}$ or $\beta=-\tau \gamma_{j k l}^{2}$ is a zero of $f_{j k l}(\beta)$ if and only if $\alpha_{j k}=0$. In the case of $\alpha_{j k}^{2}=0$, the zeros of $f_{00 l}$ are $-\tau \gamma_{00 l}^{2}$ $\left(\beta_{00 l 1}\right),-\gamma_{00 l}^{2}\left(\beta_{00 l 2}\right)$ and $-\sigma \gamma_{00 l}^{2}\left(\beta_{00 l 3}\right)$. The corresponding eigenvectors are

$$
\begin{array}{ll}
\psi^{\beta_{00 l 1}}(x, y, z)=(0,0,0,0, \sin l \pi z)^{t}, & \psi^{\beta_{00 l 2}}(x, y, z)=(0,0,0, \sin l \pi z, 0)^{t} \\
\psi_{1}^{\beta_{00 l \sigma}}(x, y, z)=(\cos l \pi z, 0,0,0,0)^{t}, & \psi_{2}^{\beta_{00 l \sigma}}(x, y, z)=(0, \cos l \pi z, 0,0,0)^{t} .
\end{array}
$$


To analyze the structure of the eigenspaces of (4.1), for $k \in \mathbb{Z}, j \in\{0\} \cup \mathbb{N}$ and $l \in \mathbb{N}$, we define

$$
\begin{aligned}
& \phi_{j k l}^{1}=\left(\frac{j \alpha_{1} l \pi}{\alpha_{j k}^{2}} \cos \left(j \alpha_{1} x+k \alpha_{2} y\right) \cos l \pi z, \frac{k \alpha_{2} l \pi}{\alpha_{j k}^{2}} \cos \left(j \alpha_{1} x+k \alpha_{2} y\right) \cos l \pi z,\right. \\
&\left.\quad \sin \left(j \alpha_{1} x+k \alpha_{2} y\right) \sin l \pi z, 0,0\right)^{t}, \\
& \phi_{j k l}^{2}=\left(0,0,0, \sin \left(j \alpha_{1} x+k \alpha_{2} y\right) \sin l \pi z, 0\right)^{t}, \\
& \phi_{j k l}^{3}=\left(0,0,0,0, \sin \left(j \alpha_{1} x+k \alpha_{2} y\right) \sin l \pi z\right)^{t}, \\
& \phi_{j k l}^{4}=\left(-\frac{j \alpha_{1} l \pi}{\alpha_{j k}^{2}} \sin \left(j \alpha_{1} x+k \alpha_{2} y\right) \cos l \pi z,-\frac{k \alpha_{2} l \pi}{\alpha_{j k}^{2}} \sin \left(j \alpha_{1} x+k \alpha_{2} y\right) \cos l \pi z,\right. \\
&\left.\quad \cos \left(j \alpha_{1} x+k \alpha_{2} y\right) \sin l \pi z, 0,0\right)^{t},
\end{aligned}
$$

The following lemma follows from (4.1)-(4.7).

Lemma 4.6. If $j^{2}+k^{2} \neq 0$ and $\beta_{j k l q}(q=1,2,3)$ is a zero of $f_{j k l}$, then we have the followings.

(1) The eigenvector corresponding to $\beta_{j k l q}$ in the complexified space of $H$ is

$$
\begin{aligned}
& \psi^{\beta_{j k l q}}=e^{i\left(j \alpha_{1} x+k \alpha_{2} y\right)}\left(\frac{i j \alpha_{1} l \pi}{\alpha_{j k}^{2}} \cos l \pi z, \frac{i k \alpha_{2} l \pi}{\alpha_{j k}^{2}} \cos l \pi z, \sin l \pi z\right. \\
&\left.A_{1}\left(\beta_{j k l q}\right) \sin l \pi z, A_{2}\left(\beta_{j k l q}\right) \sin l \pi z\right)^{t}
\end{aligned}
$$

where $A_{1}\left(\beta_{j k l q}\right)=\frac{1}{\beta_{j k l q}+\gamma_{j k l}^{2}}, \quad A_{2}\left(\beta_{j k l q}\right)=\frac{1}{\beta_{j k l q}+\tau \gamma_{j k l}^{2}}$.

(2) If $\beta_{j k l q}$ is a real number, the corresponding eigenvectors are given by

$$
\begin{aligned}
& \psi_{1}^{\beta_{j k l q}}=\phi_{j k l}^{1}+A_{1}\left(\beta_{j k l q}\right) \phi_{j k l}^{2}+A_{2}\left(\beta_{j k l q}\right) \phi_{j k l}^{3} \\
& \psi_{2}^{\beta_{j k l q}}=\phi_{j k l}^{4}+A_{1}\left(\beta_{j k l q}\right) \phi_{j k l}^{5}+A_{2}\left(\beta_{j k l q}\right) \phi_{j k l}^{6}
\end{aligned}
$$

(3) If $\operatorname{Im}\left(\beta_{j k l q}\right) \neq 0$, the generalized eigenvectors corresponding to $\beta_{j k l q}$ and $\bar{\beta}_{j k l q}$ are

$$
\begin{aligned}
& \psi_{1}^{\beta_{j k l q}}=\phi_{j k l}^{1}+R_{1}\left(\beta_{j k l q}\right) \phi_{j k l}^{2}+R_{2}\left(\beta_{j k l q}\right) \phi_{j k l}^{3}+I_{1}\left(\beta_{j k l q}\right) \phi_{j k l}^{5}+I_{2}\left(\beta_{j k l q}\right) \phi_{j k l}^{6}, \\
& \psi_{2}^{\beta_{j k l q}}=-I_{1}\left(\beta_{j k l q}\right) \phi_{j k l}^{2}-I_{2}\left(\beta_{j k l q}\right) \phi_{j k l}^{3}+\phi_{j k l}^{4}+R_{1}\left(\beta_{j k l q}\right) \phi_{j k l}^{5}+R_{2}\left(\beta_{j k l q}\right) \phi_{j k l}^{6}, \\
& \psi_{1}^{\bar{\beta}_{j k l q}}=\phi_{j k l}^{1}+R_{1}\left(\bar{\beta}_{j k l q}\right) \phi_{j k l}^{2}+R_{2}\left(\bar{\beta}_{j k l q}\right) \phi_{j k l}^{3}+I_{1}\left(\bar{\beta}_{j k l q}\right) \phi_{j k l}^{5}+I_{2}\left(\bar{\beta}_{j k l q}\right) \phi_{j k l}^{6}, \\
& \psi_{2}^{\bar{\beta}_{j k l q}}=-I_{1}\left(\bar{\beta}_{j k l q}\right) \phi_{j k l}^{2}-I_{2}\left(\bar{\beta}_{j k l q}\right) \phi_{j k l}^{3}+\phi_{j k l}^{4}+R_{1}\left(\bar{\beta}_{j k l q}\right) \phi_{j k l}^{5}+R_{2}\left(\bar{\beta}_{j k l q}\right) \phi_{j k l}^{6}, \\
& \text { where } R_{1}(\beta)=\operatorname{Re}\left(A_{1}(\beta)\right), I_{1}(\beta)=\operatorname{Im}\left(A_{1}(\beta)\right), R_{2}(\beta)=\operatorname{Re}\left(A_{2}(\beta)\right) \text { and } \\
& I_{2}(\beta)=\operatorname{Im}\left(A_{2}(\beta)\right) .
\end{aligned}
$$

Definition 4.7. (1) If $j=k=0$, for each $l \in \mathbb{N}$, we define $E_{00 l}=\operatorname{span}\left\{\psi^{\beta_{00 l 1}}, \psi^{\beta_{00 l 2}}\right\}$ and $E_{00 l}^{\sigma}=\operatorname{span}\left\{\psi_{1}^{\beta_{00 l \sigma}}, \psi_{2}^{\beta_{00 l \sigma}}\right\}$.

(2) For $j^{2}+k^{2} \neq 0$, we define $E_{j k l}^{1}=\operatorname{span}\left\{\phi_{j k l}^{1}, \phi_{j k l}^{2}, \phi_{j k l}^{3}\right\}, E_{j k l}^{2}=\operatorname{span}\left\{\phi_{j k l}^{4}, \phi_{j k l}^{5}, \phi_{j k l}^{6}\right\}$, $E_{j k l}=E_{j k l}^{1} \oplus E_{j k l}^{2}$, and $E_{j k l}^{\sigma}=\operatorname{span}\left\{\psi_{1}^{\beta_{j k l \sigma}}, \psi_{2}^{\beta_{j k l \sigma}}\right\}$. 
(3) For $(j, k, l) \in \mathbb{Z}^{2} \times \mathbb{N}$, we define $E_{f_{j k l}}$ to be the eigenspace spanned by the eigenvectors and the generalized eigenvectors corresponding to the zeros of $f_{j k l}$.

Remark 4.8. (1) It is easy to see from the Fourier expansion that $\left\{E_{j k l} \cup\right.$ $\left.E_{j k l}^{\sigma}\right\}_{j, l=0, k=-\infty}^{\infty}$ is a basis of $H_{1}$.

(2) $E_{j k l}$ (resp., $\left.E_{j k l}^{\sigma}\right)$ is orthogonal to $E_{j_{1} k_{1} l_{1}}\left(\operatorname{resp} ., E_{j_{1} k_{1} l_{1}}^{\sigma}\right)$ for $(j, k, l) \neq$ $\left(j_{1}, k_{1}, l_{1}\right)$, and $E_{j k l}$ is always orthogonal to $E_{j_{1} k_{2} l_{2}}^{\sigma}$.

The following theorem together with Lemmas 4.1 and 4.3 complete the analysis of the eigenvalue problem (4.1).

Theorem 4.9. Under the assumption 4.10), we have

1) $E_{f_{j k l}}=E_{j k l}$ for $j \in\{0\} \cup \mathbb{N}, k \in \mathbb{Z}$, and $l \in \mathbb{N}$; and

2) $L_{\lambda \mu} \mid E_{j k l}$ is strictly negative definite for each $(j, k, l) \in \mathbb{Z}^{2} \times \mathbb{N}$ when $\lambda<\lambda_{c}$.

Proof. The first assertion follows by the fact that $E_{j k l}$ is an invariant subspace of $L_{\lambda \eta}$ and $f_{j k l}$ is the characteristic polynomial of $\left.L_{\lambda \eta}\right|_{E_{j k l}}$. Assertion 1), Lemma4.3 together with the fact that $f_{j k l}$ does not have a zero of multiplicity three imply Assertion 2).

We conclude the above analysis as follows..

(1) The eigenvalues of $L_{\lambda \eta}: H_{1} \rightarrow H$ consist of $\left\{\beta_{j k l q}, \beta_{j k l \sigma}\right\}_{j, l=0, k=-\infty}^{\infty}$.

(2) The (generalized) eigenvectors of $L_{\lambda \eta}$ form a basis of $H$.

(3) $-L_{\lambda \eta}$ is a sectorial operator.

(4) For the rectangle case, the multiplicity of the first eigenvalue, $\beta_{1011}(\lambda)$, is 2 , and the corresponding eigenvectors are $\psi_{1}^{\beta_{1011}}$ and $\psi_{2}^{\beta_{1011}}$.

(5) For the square case, the multiplicity of the first eigenvalue, $\beta_{1111}(\lambda)\left(=\beta_{1-111}(\lambda)\right)$, is 4 , and the corresponding eigenvectors are $\psi_{1}^{\beta_{1111}}, \psi_{2}^{\beta_{1111}}, \psi_{1}^{\beta_{1-111}}$ and $\psi_{2}^{\beta_{1-111}}$.

To show that $G$ satisfies condition (3.3). We assume $\frac{3}{4}<\mu<1$, then for $\psi \in H_{\mu} \subset H$, by Sobolev's inequality,

$$
|G(\psi)|_{H}^{2} \leq \int_{0}^{1} \int_{0}^{2 \pi / \alpha_{2}} \int_{0}^{2 \pi / \alpha_{1}}|\psi|^{2}|\nabla \psi|^{2} d x d y d z \leq|\psi|_{L^{\infty}}^{2}|\psi|_{H_{1 / 2}}^{2} \leq C|\psi|_{H_{\mu}}^{4} .
$$

where $C$ is some constant. Hence, $G(\psi)=o\left(|\psi|_{H_{\mu}}\right)$.

4.3. Dual Basis. Since $E_{j k l}$ is finite dimensional for each $(j, k, l)$, there exists a vector $\Psi_{p}^{\beta_{j k l q}} \in E_{j k l}(q=1,2,3$ and $p=1,2)$ such that

$$
<\Psi_{p}^{\beta_{j k l q}}, \psi_{p^{*}}^{\beta_{j k l q^{*}}}>_{H}\left\{\begin{array}{lll}
\neq 0 & \text { if } & \left(q^{*}, p^{*}\right)=(q, p), \\
=0 & \text { if } & \left(q^{*}, p^{*}\right) \neq(q, p) .
\end{array}\right.
$$

We choose

$$
\Psi_{p}^{\beta_{j k l \sigma}}=\psi_{p}^{\beta_{j k l \sigma}} .
$$

Hence, by the orthogonality of $E_{j k l}$ and $E_{j k l}^{\sigma},\left\{\Psi_{p}^{\beta_{j k l q}}, \Psi_{p}^{\beta_{j k l \sigma}}\right\}_{j, l=0, k=-\infty}^{\infty}$ form a dual basis of $\mathrm{H}$ corresponding to $\left\{E_{j k l}, E_{j k l}^{\sigma}\right\}_{j, l=0, k=-\infty}^{\infty}$ in the sense that

$$
<\Psi_{p}^{\beta}, \psi_{p^{*}}^{\beta^{*}}>_{H}\left\{\begin{array}{lll}
\neq 0 & \text { if } \quad\left(\beta^{*}, p^{*}\right)=(\beta, p), \\
=0 & \text { if } \quad\left(\beta^{*}, p^{*}\right) \neq(\beta, p) .
\end{array}\right.
$$


The proof of Lemma 4.5 implies that all but finitely many of $f_{j k l}$ have three distinct real zeros. For such $f_{j k l}$ with $j^{2}+k^{2} \neq 0, \Psi_{1}^{\beta_{j k l q}}$ and $\Psi_{2}^{\beta_{j k l q}}$ could be chosen as

$$
\left\{\begin{aligned}
\Psi_{1}^{\beta_{j k l q}} & =\phi_{j k l}^{1}+C_{1}\left(\beta_{j k l q}\right) \phi_{j k l}^{2}+C_{2}\left(\beta_{j k l q}\right) \phi_{j k l}^{3} \\
\Psi_{2}^{\beta_{j k l q}} & =\phi_{j k l}^{4}+C_{1}\left(\beta_{j k l q}\right) \phi_{j k l}^{5}+C_{2}\left(\beta_{j k l q}\right) \phi_{j k l}^{6}
\end{aligned}\right.
$$

where

$$
\left\{\begin{array}{l}
C_{1}\left(\beta_{j k l q}\right)=\frac{\sigma \lambda}{\beta_{j k l q}+\gamma_{j k l}^{2}}=\sigma \lambda A_{1}\left(\beta_{j k l q}\right) \\
C_{2}\left(\beta_{j k l q}\right)=\frac{-\sigma \eta}{\beta_{j k l q}+\tau \gamma_{j k l}^{2}}=-\sigma \eta A_{2}\left(\beta_{j k l q}\right)
\end{array}\right.
$$

\section{The Proofs of the Main Theorems}

We are now in a position to reduce equations of (2.1)- (2.4) to the center manifold. We would like to fix $\eta<\eta_{c}$, and let $\lambda \approx \lambda_{c}$ be the bifurcation parameter. For any $\psi=(U, T, S) \in H$, we have

$$
\psi=\sum_{k=-\infty}^{\infty} \sum_{j=0, l=1}^{\infty} \sum_{q=1}^{3}\left(x_{j k l q} \psi_{1}^{\beta_{j k l q}}+y_{j k l q} \psi_{2}^{\beta_{j k l q}}\right)+\sum_{k=-\infty}^{\infty} \sum_{j, l=0}^{\infty}\left(x_{j k l \sigma} \psi_{1}^{\beta_{j k l \sigma}}+y_{j k l \sigma} \psi_{2}^{\beta_{j k l \sigma}}\right) .
$$

For the square case, we assume $\alpha_{1}=\alpha_{2}=\frac{\pi}{2}$. Hence $\beta_{1111}(\lambda)=\beta_{1-111}(\lambda)$ are the first eigenvalues.

For brevity, we let $\beta_{0}(\lambda)=\beta_{1111}(\lambda)=\beta_{1-111}(\lambda)$, then the reduced equations are given by

$$
\left\{\begin{array}{l}
\left.\frac{d x_{1111}}{d t}=\beta_{0}(\lambda) x_{1111}+\frac{1}{<\psi_{1}^{\beta_{1111}}, \Psi_{1}^{\beta_{1111}}>_{H}}<G(\psi, \psi), \Psi_{1}^{\beta_{1111}}\right)>_{H}, \\
\left.\frac{d y_{1111}}{d t}=\beta_{0}(\lambda) y_{1111}+\frac{1}{<\psi_{2}^{\beta_{1111}}, \Psi_{2}^{\beta_{1111}}>_{H}}<G(\psi, \psi), \Psi_{2}^{\beta_{1111}}\right)>_{H}, \\
\left.\frac{d x_{1-111}}{d t}=\beta_{0}(\lambda) x_{1-111}+\frac{1}{<\psi_{1}^{\beta_{1-111}}, \Psi_{1}^{\beta_{1-111}}>_{H}}<G(\psi, \psi), \Psi_{1}^{\beta_{1-111}}\right)>_{H}, \\
\left.\frac{d y_{1-111}}{d t}=\beta_{0}(\lambda) y_{1-111}+\frac{1}{<\psi_{2}^{\beta_{1-111}}, \Psi_{2}^{\beta_{1-111}}>_{H}}<G(\psi, \psi), \Psi_{2}^{\beta_{1-111}}\right)>_{H} .
\end{array}\right.
$$

Here for $\psi_{1}=\left(U_{1}, T_{1}, S_{1}\right), \psi_{2}=\left(U_{2}, T_{2}, S_{2}\right)$ and $\psi_{3}=\left(U_{3}, T_{3}, S_{3}\right)$,

$$
\begin{aligned}
<G\left(\psi_{1}, \psi_{2}\right), \psi_{3}>_{H}= & -\int_{0}^{1} \int_{0}^{2 \pi / \alpha_{2}} \int_{0}^{2 \pi / \alpha_{1}}\left[<\left(U_{1} \cdot \nabla\right) U_{2}, U_{3}>_{\mathbb{R}^{3}}\right. \\
& \left.+\left(U_{1} \cdot \nabla\right) T_{2} T_{3}+\left(U_{1} \cdot \nabla\right) S_{2} S_{3}\right] d x d y d z .
\end{aligned}
$$

Let the center manifold function be denoted by

$\Phi=\sum_{\beta \neq \beta_{1111}, \beta_{1-111}}\left(\Phi_{1}^{\beta}\left(x_{1111}, y_{1111}, x_{1-111}, y_{1-111}\right) \psi_{1}^{\beta}+\Phi_{2}^{\beta}\left(x_{111}, y_{111}, x_{1-111}, y_{1-111}\right) \psi_{2}^{\beta}\right)$.

Note that for any $\psi_{i} \in H_{1}(i=1,2,3),<G\left(\psi_{1}, \psi_{2}\right), \psi_{2}>_{H}=0,<G\left(\psi_{1}, \psi_{2}\right), \psi_{3}>_{H}=$ $-<G\left(\psi_{1}, \psi_{3}\right), \psi_{2}>_{H}$, and for any $\psi_{i} \in E_{j k l}(i=1,2,3),<G\left(\psi_{1}, \psi_{2}\right), \psi_{3}>_{H}=0$. 
Applying Theorem 3.8 in [1], we obtain

$$
\begin{aligned}
\Phi^{\beta_{0021}} & =\frac{A_{2} \pi}{2 \beta_{0021}}\left[x_{1111}^{2}+y_{1111}^{2}+x_{1-111}^{2}+y_{1-111}^{2}\right]+o(2), \\
\Phi^{\beta_{0022}} & =\frac{A_{1} \pi}{2 \beta_{0022}}\left[x_{1111}^{2}+y_{1111}^{2}+x_{1-111}^{2}+y_{1-111}^{2}\right]+o(2), \\
\Phi_{1}^{\beta_{202 q}} & =B\left(\beta_{202 q}\right)\left(x_{1111} y_{1-111}+x_{1-111} y_{1111}\right)+o(2), \\
\Phi_{2}^{\beta_{202 q}} & =B\left(\beta_{202 q}\right)\left(-x_{1111} x_{1-111}+y_{1111} y_{1-111}\right)+o(2), \\
\Phi_{1}^{\beta_{022 q}} & =B\left(\beta_{022 q}\right)\left(x_{1111} y_{1-111}-x_{1-111} y_{1111}\right)+o(2), \\
\Phi_{2}^{\beta_{022 q}} & =B\left(\beta_{022 q}\right)\left(x_{1111} x_{1-111}+y_{1111} y_{1-111}\right)+o(2),
\end{aligned}
$$

$$
\begin{aligned}
& \Phi\left(x_{1111}, y_{1111}, x_{1-111}, y_{1-111}\right)=\Phi^{\beta_{0021}} \psi^{\beta_{0021}}+\Phi^{\beta_{0022}} \psi^{\beta_{0022}} \\
& +\sum_{q=1}^{3}\left(\Phi_{1}^{\beta_{202 q}} \psi_{1}^{\beta_{202 q}}+\Phi_{2}^{\beta_{202 q}} \psi_{2}^{\beta_{202 q}}+\Phi_{1}^{\beta_{022 q}} \psi_{1}^{\beta_{022 q}}+\Phi_{2}^{\beta_{022 q}} \psi_{2}^{\beta_{022 q}}\right)+o(2),
\end{aligned}
$$

where

$$
\begin{aligned}
B(\beta)= & \frac{\pi\left(3+A_{1}\left(\beta_{0}\right) C_{1}(\beta)+A_{2}\left(\beta_{0}\right) C_{2}(\beta)\right)}{2 \beta\left(5+A_{1}(\beta) C_{1}(\beta)+A_{2}(\beta) C_{2}(\beta)\right)}, \\
C_{1}(\beta)= & \frac{\sigma \lambda}{\left(\beta+\gamma_{202}^{2}\right)}, \quad C_{2}(\beta)=\frac{\sigma \eta}{\left(\beta+\tau \gamma_{202}^{2}\right)}, \\
o(2)= & o\left(x_{1111}^{2}+y_{1111}^{2}+x_{1-111}^{2}+y_{1-111}^{2}\right) \\
& +O\left(\left|\beta_{0}(\lambda)\right| \cdot\left(x_{1111}^{2}+y_{1111}^{2}+x_{1-111}^{2}+y_{1-111}^{2}\right)\right) .
\end{aligned}
$$

Hereafter, we make the following convention,

$$
\begin{aligned}
o(3)= & o\left(\left(x_{1111}^{2}+y_{1111}^{2}+x_{1-111}^{2}+y_{1-111}^{2}\right)^{3 / 2}\right) \\
& +O\left(\left|\beta_{0}(\lambda)\right| \cdot\left(x_{1111}^{2}+y_{1111}^{2}+x_{1-111}^{2}+y_{1-111}^{2}\right)^{3 / 2}\right), \\
o(4)= & o\left(\left(x_{1111}^{2}+y_{1111}^{2}+x_{1-111}^{2}+y_{1-111}^{2}\right)^{2}\right) \\
& +O\left(\left|\beta_{0}(\lambda)\right| \cdot\left(x_{1111}^{2}+y_{1111}^{2}+x_{1-111}^{2}+y_{1-111}^{2}\right)^{2}\right) .
\end{aligned}
$$

By (5.3) and the fact that $\beta_{022 q}=\beta_{202 q}$, we obtain

$$
\begin{aligned}
<G(\psi, \psi), \Psi_{1}^{\beta_{1111}}>_{H}= & <G\left(\psi_{1}^{\beta_{1111}}, \Phi\right), \Psi_{1}^{\beta_{1111}}>_{H}+<G\left(\psi_{2}^{\beta_{1111}}, \Phi\right), \Psi_{1}^{\beta_{1111}}>_{H} \\
& \left.+<G\left(\psi_{1}^{\beta_{1-111}}, \Phi\right), \Psi_{1}^{\beta_{1111}}>_{H}+<G\left(\psi_{2}^{\beta_{1-111}}\right), \Phi\right), \Psi_{1}^{\beta_{1111}}>_{H}+o(3) \\
= & -<G\left(\psi_{1}^{\beta_{1111}}, \Psi_{1}^{\beta_{1111}}\right), \Phi>_{H}-<G\left(\psi_{2}^{\beta_{1111}}, \Psi_{1}^{\beta_{1111}}\right), \Phi>_{H} \\
& \left.-<G\left(\psi_{1}^{\beta_{1-111}}, \Psi_{1}^{\beta_{1111}}\right), \Phi>_{H}-<G\left(\psi_{2}^{\beta_{1-111}}\right), \Psi_{1}^{\beta_{1111}}\right), \Phi>_{H}+o(3) \\
= & 2 \pi^{2}\left(\frac{A_{1} C_{1}}{\beta_{0022}}+\frac{A_{2} C_{2}}{\beta_{0021}}\right)\left(x_{1111}^{2}+y_{1111}^{2}+x_{1-111}^{2}+y_{1-111}^{2}\right) x_{1111} \\
& +2 \sum_{q=1}^{3} B^{*}\left(\beta_{202 q}\right) B\left(\beta_{202 q}\right)\left(x_{1-111}^{2}+y_{1-111}^{2}\right) x_{1111}+o(3),
\end{aligned}
$$

where

$$
B^{*}(\beta)=\pi\left(3+A_{1}(\beta) C_{1}\left(\beta_{0}\right)+A_{2}(\beta) C_{2}\left(\beta_{0}\right)\right) .
$$


Similarly, we derive that

$$
\begin{aligned}
<G(\psi, \psi), \Psi_{2}^{\beta_{1111}}>_{H}= & 2 \pi^{2}\left(\frac{A_{1} C_{1}}{\beta_{0022}}+\frac{A_{2} C_{2}}{\beta_{0021}}\right)\left(x_{1111}^{2}+y_{1111}^{2}+x_{1-111}^{2}+y_{1-111}^{2}\right) y_{1111} \\
& +2 \sum_{q=1}^{3} B^{*}\left(\beta_{202 q}\right) B\left(\beta_{202 q}\right)\left(x_{1-111}^{2}+y_{1-111}^{2}\right) y_{1111}+o(3), \\
<G(\psi, \psi), \Psi_{1}^{\beta_{1-111}}>_{H}= & 2 \pi^{2}\left(\frac{A_{1} C_{1}}{\beta_{0022}}+\frac{A_{2} C_{2}}{\beta_{0021}}\right)\left(x_{1111}^{2}+y_{1111}^{2}+x_{1-111}^{2}+y_{1-111}^{2}\right) x_{1-111} \\
& +2 \sum_{q=1}^{3} B^{*}\left(\beta_{202 q}\right) B\left(\beta_{202 q}\right)\left(x_{1111}^{2}+y_{1111}^{2}\right) x_{1-111}+o(3), \\
<G(\psi, \psi), \Psi_{2}^{\beta_{1-111}}>_{H}= & 2 \pi^{2}\left(\frac{A_{1} C_{1}}{\beta_{0022}}+\frac{A_{2} C_{2}}{\beta_{0021}}\right)\left(x_{1111}^{2}+y_{1111}^{2}+x_{1-111}^{2}+y_{1-111}^{2}\right) y_{1-111} \\
& +2 \sum_{q=1}^{3} B^{*}\left(\beta_{202 q}\right) B\left(\beta_{202 q}\right)\left(x_{1111}^{2}+y_{1111}^{2}\right) y_{1-111}+o(3) .
\end{aligned}
$$

Therefore, (5.1) can be rewritten as

$$
\left\{\begin{array}{l}
\frac{d x_{1111}}{d t}=\beta_{0}(\lambda) x_{1111}+\delta_{1} E x_{1111}+\delta_{2}\left(x_{1-111}^{2}+y_{1-111}^{2}\right) x_{1111}+o(3) \\
\frac{d y_{1111}}{d t}=\beta_{0}(\lambda) y_{1111}+\delta_{1} E y_{1111}+\delta_{2}\left(x_{1-111}^{2}+y_{1-111}^{2}\right) y_{1111}+o(3) \\
\frac{d x_{1-111}}{d t}=\beta_{0}(\lambda) x_{1-111}+\delta_{1} E x_{1-111}+\delta_{2}\left(x_{1111}^{2}+y_{1111}^{2}\right) x_{1-111}+o(3) \\
\frac{d y_{1-111}}{d t}=\beta_{0}(\lambda) y_{1-111}+\delta_{1} E y_{1-111}+\delta_{2}\left(x_{1111}^{2}+y_{1111}^{2}\right) y_{1-111}+o(3)
\end{array}\right.
$$

where $E=\left(x_{1111}^{2}+y_{1111}^{2}+x_{1-111}^{2}+y_{1-111}^{2}\right)$,

$$
\begin{aligned}
\delta_{1} & =\delta_{1}(\lambda, \eta)=2 \pi^{2}\left(\frac{A_{1} C_{1}}{\beta_{0022}}+\frac{A_{2} C_{2}}{\beta_{0021}}\right) / 4\left(3+A_{1} C_{1}+A_{2} C_{2}\right) \\
& =-\frac{\left(A_{1} C_{1}+\tau^{-1} A_{2} C_{2}\right)}{8\left(3+A_{1} C_{1}+A_{2} C_{2}\right)}, \\
\delta_{2} & =\delta_{2}(\lambda, \eta)=2 \sum_{q=1}^{3} B^{*}\left(\beta_{202 q}\right) B\left(\beta_{202 q}\right) / 4\left(3+A_{1} C_{1}+A_{2} C_{2}\right) .
\end{aligned}
$$

The energy estimate of (5.6) is given by

$$
\frac{1}{2} \frac{d E}{d t}=\beta_{0}(\lambda) E+\delta_{1} E^{2}+2 \delta_{2}\left(x_{1111}^{2}+y_{1111}^{2}\right)\left(x_{1-111}^{2}+y_{1-111}^{2}\right)+o(4) .
$$

If the quadratic form

$$
\delta_{1} E^{2}+2 \delta_{2}\left(x_{1111}^{2}+y_{1111}^{2}\right)\left(x_{1-111}^{2}+y_{1-111}^{2}\right),
$$

is negative definite at $\lambda=\lambda_{c}$, we conclude that $(U, T, S)=0$ is a locally asymptotically stable equilibrium point of (2.1)-(2.4). We then obtain Theorem 3.5 by Theorem 3.1 directly. And if the quadratic form (5.10) is positive definite, we obtain the subcritical bifurcation. This completes the proofs of Theorem 3.5 and Theorem 3.6 . 
For the rectangle case, the first eigenspace is of dimension two. The reduced equations on the center manifold are given by

$$
\left\{\begin{array}{l}
\frac{d x_{1011}}{d t}=\beta_{1011}(\lambda) x_{1011}+\delta_{1}\left(x_{1011}^{2}+y_{1011}^{2}\right) x_{1011}+o(3), \\
\frac{d y_{1011}}{d t}=\beta_{1011}(\lambda) y_{1011}+\delta_{1}\left(x_{1011}^{2}+y_{1011}^{2}\right) y_{1011}+o(3) .
\end{array}\right.
$$

The rest part of the proofs of Theorem 3.2. Theorem 3.3 and Theorem 3.4 is similar to the $2 \mathrm{D}$ case in [8]. In this case, we can use Theorem 5.10 in 13 or invariant sphere theorem in [4] to conclude the $S^{1}$ structure of the bifurcating solution.

Remark 5.1. (1) As we have already proved in [8], we know that

$$
\delta_{1}\left\{\begin{array}{lll}
<0 & \text { if } & \eta<\eta_{c_{1}} \\
>0 & \text { if } & \eta>\eta_{c_{1}}
\end{array} .\right.
$$

(2) When $\sigma, \tau, \lambda$ and $\eta$ are given, $\delta_{1}$ and $\delta_{2}$ can be evaluated numerically.

Acknowledgements. The authors would like to thank the referee's insightful comments and suggestions. The work was supported in part by the Office of Naval Research, by the National Science Foundation, and by the National Science Foundation of China.

\section{REFERENCES}

[1] P. G. Baines And A. Gill, On thermohaline convection with linear gradients, J. Fluid Mech., 37 (1969), pp. 289-306.

[2] F. H. Busse, On the stability of two-dimensional convection in a layer heated from below, J. Math.\& Phys., 46 (1976), pp. 140-143.

[3] E. Buzano and M. Golubitsky, Bifurcation on the hexagonal lattice and the planar Bénard problem, Philos. Trans. Roy. Soc. London Ser. A, 308 (1983), pp. 617-667.

[4] M. Field, Equivariant bifurcation theory and symmetry breaking, J. Dynam. Differential Equations, 1 (1989), pp. 369-421.

5] C. Foias, O. Manley, and R. Temam, Attractors for the Bénard problem: existence and physical bounds on their fractal dimension, Nonlinear Anal., 11 (1987), pp. 939-967.

[6] M. Golubitsky, I. Stewart, and D. G. Schaeffer, Singularities and groups in bifurcation theory. Vol. II, vol. 69 of Applied Mathematical Sciences, Springer-Verlag, New York, 1988.

[7] D. Henry, Geometric theory of semilinear parabolic equations, vol. 840 of Lecture Notes in Mathematics, Springer-Verlag, Berlin, 1981.

[8] C.-H. Hsia, T. MA, AND S. WANG, Bifurcation and stability of two-dimensional doublediffusive convection, submitted, (2006).

[9] W. S. Jevons, On the cirrous form of cloud, London, Edinburgh, Dublin Philos. Mag. J. Sci. Ser. 4, 14 (1857), pp. 22-35.

[10] J. L. Lions, R. Temam, And S. WAng, On the equations of large-scale ocean, Nonlinearity, 5 (1992), pp. 1007-1053.

[11] T. Ma And S. WAng, Bifurcation Theory and Applications, vol. 53 of World Scientific Series on Nonlinear Science, Series A, World Scientific, 2005.

[12] — Dynamic bifurcation of nonlinear evolution equations and applications, Chinese Annals of Mathematics, 26:2 (2005), pp. 185-206.

[13] - Geometric Theory of Incompressible Flows with Applications to Fluid Dynamics, vol. 119 of Mathematical Surveys and Monographs, American Mathematical Society, Providence, RI, 2005.

[14] W. Nagata and J. W. Thomas, Bifurcation in double-diffusive systems i. equilibrium solutions, SJMA, 17:1 (1986), pp. 91-113.

[15] D. H. Sattinger, Group representation theory, bifurcation theory and pattern formation, J. Funct. Anal., 28 (1978), pp. 58-101. 
[16] M. E. Stern, The "salt fountain" and thermohaline convection, Tellus, 12 (1960), pp. 172175.

[17] H. Stommel, A. Arons, and D. Blanchard, An oceanographical curiosity : the perpetual salt fountain, Deep-Sea Res., 3 (1956), pp. 152-153.

[18] R. Tемам, Infinite-dimensional dynamical systems in mechanics and physics, vol. 68 of Applied Mathematical Sciences, Springer-Verlag, New York, second ed., 1997.

[19] G. Veronis, On finite amplitude instability in the thermohaline convection, J. Marine Res., 23 (1965), pp. 1-17.

(CH) Department of Mathematics, Indiana University, Bloomington, IN 47405

E-mail address: chsia@indiana.edu

(TM) Department of Mathematics, Sichuan University, Chengdu, P. R. China

(SW) Department of Mathematics, Indiana University, Bloomington, IN 47405

E-mail address: showang@indiana.edu 